PROCEEDINGS OF THE

AMERICAN MATHEMATICAL SOCIETY

Volume 131, Number 12, Pages 3737-3746

S 0002-9939(03)06950-8

Article electronically published on February 20, 2003

\title{
INNER BOUNDS FOR THE SPECTRUM OF QUASINORMAL OPERATORS
}

\author{
M. I. GIL
}

(Communicated by Joseph A. Ball)

\begin{abstract}
A linear operator in a separable Hilbert space is called a quasinormal one if it is a sum of a normal operator and a compact one. In the paper, bounds for the spectrum of quasinormal operators are established. In addition, the lower estimate for the spectral radius is derived. Under some restrictions, that estimate improves the well-known results. Applications to integral operators and matrices are discussed. Our results are new even in the finite-dimensional case.
\end{abstract}

\section{InTRODUCTION AND STATEMENT OF THE MAIN RESUlT}

Many papers and books are devoted to the spectrum of linear operators. Mainly, the asymptotic distributions of the eigenvalues are considered; cf. the books by König [Ko, Pietsch [Pi], and references therein. However, in many applications, for example, in numerical mathematics and stability analysis, bounds for eigenvalues are very important. But the bounds are investigated considerably less than the asymptotic distributions.

Let $H$ be a separable Hilbert space with a scalar product (., .), the norm $\|$.$\| and$ the unit operator $I$. For a linear operator $A, \sigma(A)$ is the spectrum, $\operatorname{Dom}(A)$ is the domain, $r_{s}(A) \equiv \sup |\sigma(A)|$ is the (upper) spectral radius, and $r_{l}(A) \equiv \inf |\sigma(A)|$ is the inner (lower) spectral radius. In addition, $\alpha(A) \equiv \sup \operatorname{Re} \sigma(A)$.

A linear operator in $H$ is called a quasinormal one if it is a sum of a normal operator and a compact one. In the present paper, for a class of quasinormal operators, lower bounds for $r_{s}(A), \alpha(A)$ and upper bounds for $r_{l}(A)$ are derived. They are new even in the finite-dimensional case. In addition, applications of these bounds to matrices and integral operators are discussed.

Note that lower estimates for $r_{s}(A)$ for positive (finite and infinite) matrices and integral operators are well known [MM], $[\mathrm{Kr}]$. But in the case of operators which are not positive, in general, to the best of our knowledge, the lower estimates for $r_{s}(A)$ were not investigated. At the same time our estimates below are also valid for non-positive matrices and integral operators. Moreover, in the case of positive matrices and integral operators, under some restrictions below, we improve the well-known results, in particular, the Frobenius estimate.

Received by the editors March 21, 2001 and, in revised form, June 24, 2002

2000 Mathematics Subject Classification. Primary 47A55, 47A75; Secondary 47G10, $47 \mathrm{G} 20$.

Key words and phrases. Linear operators, spectrum, spectral radius, integral operators, finite and infinite matrices.

This research was supported by the Israel Ministry of Science and Technology. 
Recall that a linear operator $V$ is a Volterra one if it is quasinilpotent (that is, $\sigma(V)=\{0\})$ and compact; cf. the book by Gohberg and Krein [GK]. Furthermore, let $P(t)(-\infty \leq t \leq \infty)$ be a maximal resolution of the identity. That is, $P(t)$ is a continuous on the left orthogonal resolution of the identity defined on $(-\infty, \infty)$. Moreover, any gap $P\left(t_{0}+0\right)-P\left(t_{0}\right)$ of $P(t)$ (if it exists) is one-dimensional; cf. the books by Brodskii [Br], Gohberg and Krein [GK] and Gil' Gi1 p. 69].

Let us consider a linear operator $A$ in $H$ of the type

$$
A=D+V_{+}+V_{-}
$$

where $D$ is a normal (generally unbounded) operator, and $V_{-}$and $V_{+}$are Volterra operators. It is assumed that for a maximal resolution of the identity $P($.$) ,$

$$
\begin{gathered}
P(t) D h=D P(t) h, P(t) V_{+} P(t)=V_{+} P(t) \text { and } P(t) V_{-} P(t)=P(t) V_{-} \\
(h \in \operatorname{Dom}(D) ; t \in \mathbf{R}) .
\end{gathered}
$$

In addition, there is a monotonically increasing continuous scalar-valued function $\phi(z)(z \geq 0)$ with the properties $\phi(0)=0, \phi(\infty)=\infty$, such that the inequality

$$
\left\|(\lambda I-A)^{-1}\right\| \leq \phi\left(\rho^{-1}(A, \lambda)\right)
$$

holds, where $\rho(A, \lambda)$ is the distance between $\sigma(A)$ and a regular point $\lambda \in \mathbf{C}$ of $A$. Put

$$
\nu(A)=\min \left\{\left\|V_{-}\right\|,\left\|V_{+}\right\|\right\}
$$

and denote by $z(\phi)$ the unique positive root of the equation

$$
\nu(A) \phi(1 / z)=1(z \geq 0) .
$$

Now we are in a position to formulate the main result of the paper.

Theorem 1.1. Let $A$ be defined by (1.1) and let conditions (1.2) and (1.3) hold. Then for any $\mu \in \sigma(D)$ there is a $\mu_{0} \in \sigma(A)$, such that

$$
\left|\mu_{0}-\mu\right| \leq z(\phi) \text {. }
$$

The proof of this theorem is presented in the next section.

Corollary 1.2. Under the hypothesis of Theorem 1.1, the following inequalities are true:

$$
\begin{gathered}
r_{s}(A) \geq \max \left\{0, r_{s}(D)-z(\phi)\right\} \text { if } D \text { is bounded, } \\
r_{l}(A) \leq r_{l}(D)+z(\phi) \text { and } \\
\alpha(A) \geq \alpha(D)-z(\phi) \text { if } \alpha(D)<\infty .
\end{gathered}
$$

Indeed, take $\mu$ in such a way that $|\mu|=r_{s}(D)$. Then due to (1.6), there is $\mu_{0} \in \sigma(A)$, such that $\left|\mu_{0}\right| \geq r_{s}(D)-z(\phi)$. Hence, (1.7) follows. Similarly, inequality (1.8) can be proved.

Furthermore, take $\mu$ in such a way that $\operatorname{Re} \mu=\alpha(D)$. Due to (1.6) for some $\mu_{0} \in$ $\sigma(A),\left|\operatorname{Re} \mu_{0}-\alpha(D)\right| \leq z(\phi)$. So, either $\operatorname{Re} \mu_{0} \geq \alpha(D)$ or $\operatorname{Re} \mu_{0} \geq \alpha(D)-z(\phi)$. Thus, inequality (1.9) is also proved.

We will say that operator $A$ is unstable if $\alpha(A)>0$. Due to the previous corollary, under the hypothesis of Theorem 1.1, $A$ is unstable, provided $\alpha(D)-z(\phi)>0$. 


\section{Proof of Theorem 1.1}

1. Let $B$ be a linear operator in $H$. Recall that the quantity

$$
s v_{A}(B) \equiv \sup _{\mu \in \sigma(B)} \inf _{\lambda \in \sigma(A)}|\mu-\lambda|
$$

is called the spectral variation of $B$ with respect to $A$. Furthermore, let linear operators $A$ and $B$ in $H$ satisfy the conditions $\operatorname{Dom}(A)=\operatorname{Dom}(B)$ and $q_{B} \equiv$ $\|A-B\|<\infty$. In addition, assume that condition (1.3) holds. Then due to Lemma 4.1.4 Gi1], the inequality

$$
s v_{A}(B) \leq z\left(\phi, q_{B}\right)
$$

is true, where $z\left(\phi, q_{B}\right)$ is the extreme right-hand (positive) root of the equation $q_{B} \phi(1 / z)=1$. We will say that a maximal resolution of the identity $P_{0}($.$) belongs$ to a linear operator $A_{0}$ if $P_{0}(t)$ projects onto invariant subspaces of $A_{0}$ for all $t \in \mathbf{R}$. If a maximal resolution of the identity $P_{0}($.$) belongs to a normal operator D_{0}$ and to a nilpotent operator $V_{0}$, clearly it also belongs to the operator $A_{0}=D_{0}+V_{0}$ and due to Lemma 3.2.12 from Gi1,

$$
\sigma\left(A_{0}\right)=\sigma\left(D_{0}\right)
$$

2. Furthermore, thanks to $(1.2), P($.$) , belongs to operators D$ and $V_{+}$. Take $B_{+}=D+V_{+}$. Then due to $(2.2), \sigma\left(B_{+}\right)=\sigma(D)$. Relation (2.1) implies that, for any $\mu \in \sigma(D)$, there is $\mu_{0} \in \sigma(A)$, such that

$$
\left|\mu_{0}-\mu\right| \leq z_{-},
$$

where $z_{-}$is the unique positive root of the equation

$$
\left\|V_{-}\right\| \phi(1 / z)=1 \quad(z \geq 0) .
$$

Now, take $B_{-}=D+V_{-}$. Put $\tilde{P}(t)=I-P(t)$. Clearly, $\tilde{P}($.$) is a maximal resolution$ of the identity. Moreover, according to $(1.2), \tilde{P}(t) D h=D \tilde{P}(t) h(h \in D o m(D))$ and

$$
(I-\tilde{P}(t)) V_{-}(I-\tilde{P}(t))=(I-\tilde{P}(t)) V_{-} .
$$

Hence, $\tilde{P}(t) V_{-} \tilde{P}(t)=V_{-} \tilde{P}(t) \quad(t \in \mathbf{R})$. So $\tilde{P}($.$) belongs to operators D$ and $V_{-}$. Therefore, due to relation (2.2), we get $\sigma\left(B_{-}\right)=\sigma(D)$. In addition, inequality (2.1) implies that for any $\mu \in \sigma(D)$, there is $\mu_{0} \in \sigma(A)$ such that

$$
\left|\mu_{0}-\mu\right| \leq z_{+},
$$

where $z_{+}$is the unique positive root of the equation $\left\|V_{+}\right\| \phi(1 / z)=1(z \geq 0)$, since $\left\|A-B_{-}\right\|=\left\|V_{+}\right\|$. Relations (2.3) and (2.4) prove the required result.

\section{Finite-Dimensional operators}

Let $\mathbf{C}^{n}$ be an $n$-dimensional Euclidean space with the Euclidean norm $\|$.$\| . In$ this section $A=\left(a_{j k}\right)_{j, k=1}^{n}$ is an $n \times n$-matrix. Let us introduce the following quantity (Henrici's departure from normality):

$$
g(A)=\left(N^{2}(A)-\sum_{k=1}^{n}\left|\lambda_{k}(A)\right|^{2}\right)^{1 / 2},
$$


where $\lambda_{k}(A)(k=1, \ldots, n)$ are the eigenvalues taken with their multiplicities, and $N\left(\right.$.) is the Hilbert-Schmidt (Frobenius) norm $N^{2}(A)=$ Trace $A^{*} A$. The asterisk means the adjointness. As it is proved in Gi3 p. 353], for any regular $\lambda$,

$$
\left\|(\lambda I-A)^{-1}\right\| \leq \sum_{k=0}^{n-1} \frac{g^{k}(A) \gamma_{n, k}}{\rho^{k+1}(A, \lambda)} .
$$

Here

$\gamma_{n, 0}=1, \gamma_{n, p}=\left[C_{n-1}^{p}(n-1)^{-p}\right]^{1 / 2}$, where $C_{n-1}^{p}=\frac{(n-1) !}{p !(n-1-p) !} \quad(p=1, \ldots, n-1)$

are the binomial coefficients. Simple calculations show that

$$
\gamma_{n, p} \leq \frac{1}{\sqrt{p !}}(p=1, \ldots, n-1)
$$

If $A$ is a normal matrix $A A^{*}=A^{*} A$, then $g(A)=0$. Moreover,

$$
g(A) \leq \sqrt{1 / 2} N\left(A^{*}-A\right)
$$

cf. Gi1 Corollary 1.3.7].

Let $V_{+}, V_{-}$be the upper and lower triangular parts of $A$ :

$$
V_{+}=\left(\begin{array}{cccc}
0 & a_{12} & \ldots & a_{1 n} \\
0 & 0 & \ldots & a_{2 n} \\
. & . & \ldots & . \\
0 & 0 & \ldots & 0
\end{array}\right) \text { and } V_{-}=\left(\begin{array}{cccc}
0 & 0 & \ldots & 0 \\
a_{21} & 0 & \ldots & 0 \\
\cdot & . & \ldots & . \\
a_{n 1} & a_{n 2} & \ldots & 0
\end{array}\right) \text {. }
$$

Denote by $z_{n}(A)$ the unique positive root of the equation

$$
z^{n}(A)=\nu(A) \sum_{k=0}^{n-1} g^{k}(A) \gamma_{n, k} z^{n-k-1}
$$

with $\nu(A)$ defined by (1.4).

Theorem 3.1. Let $A=\left(a_{j k}\right)$ be an $n \times n$-matrix. Then for any $k=1, \ldots, n$, there is an eigenvalue $\mu_{0}$ of $A$, such that $\left|\mu_{0}-a_{k k}\right| \leq z_{n}(A)$. Moreover, the following inequalities are true:

$$
\begin{gathered}
r_{s}(A) \geq \max \left\{0, \max _{k=1, \ldots, n}\left|a_{k k}\right|-z_{n}(A)\right\}, \\
r_{l}(A) \leq \min _{k=1, \ldots, n}\left|a_{k k}\right|+z_{n}(A), \text { and } \alpha(A) \geq \max _{k=1, \ldots, n} \text { Re } a_{k k}-z_{n}(A) .
\end{gathered}
$$

Proof. Let $\left\{P_{k}\right\}_{k=1}^{n}$ be the projectors defined by

$$
P_{k} h=\text { column }\left[h_{1}, h_{2}, \ldots, h_{k}, 0, \ldots, 0\right]
$$

for an arbitrary vector $h=$ column $\left[h_{1}, h_{2}, \ldots, h_{n}\right] \in \mathbf{C}^{n}$. Then $\left\{P_{k}\right\}_{k=1}^{n}$ is the maximal resolution of the identity. Moreover, conditions (1.2) are valid, where $D$ is the main diagonal of matrix $A$, and $V_{+}$and $V_{-}$are the pointed upper and lower triangular parts.

Equation (3.2) is equivalent to the following one:

$$
\nu(A) \sum_{k=0}^{n-1} g^{k}(A) \gamma_{n, k} z^{-k-1}=1 .
$$

Now, taking into account inequality (3.1), we get the required result by virtue of Theorem 1.1 and Corollary 1.2. 
Put

$$
w_{n}(A)=\nu(A) \sum_{k=0}^{n-1} g^{k}(A) \gamma_{n, k} .
$$

Due to the trivial Lemma 1.11.1 [Gi3], $z_{n}(A) \leq \delta_{n}(A)$, where

$$
\delta_{n}(A)=\sqrt[n]{w_{n}} \text { if } w_{n}(A) \leq 1 \text { and } \delta_{n}(A)=w_{n} \text { if } w_{n}(A) \geq 1 .
$$

Now Theorem 3.1 implies

$$
\begin{gathered}
r_{s}(A) \geq \max _{k=1, \ldots, n}\left|a_{k k}\right|-\delta_{n}(A), \\
r_{l}(A) \leq \min _{k=1, \ldots, n}\left|a_{k k}\right|+\delta_{n}(A), \text { and } \alpha(A) \geq \max _{k=1, \ldots, n} \operatorname{Re} a_{k k}-\delta_{n}(A) .
\end{gathered}
$$

Recall that for non-negative matrices Frobenius has derived the following lower estimate:

$$
r_{s}(A) \geq \tilde{r}(A) \equiv \min _{j=1, \ldots, n} \sum_{k=1}^{n} a_{j k}
$$

cf. [MM, Chapter 3, Section 3.1]. Relation (3.4) improves estimate (3.5) in the case $\left|a_{j k}\right|=a_{j k}(j, k=1, \ldots, n)$ provided $\max _{k} a_{k k}-\delta_{n}(A)>\tilde{r}(A)$. That is, (3.4) is sharper than (3.5) for matrices which are close to triangular ones, since $\delta_{n}(A) \rightarrow 0$ when $V_{-} \rightarrow 0$ or $V_{+} \rightarrow 0$. It should be noted that in the cases where the largest (or rightmost, or smallest modulus) Gerschgorin circle is disjoint from the others, inequality (3.5) provides bounds that bear an even closer resemblance to inequality (3.4).

Note that in Gi2, Gi6] the new upper estimates for the spectral radius of matrices were established. They improve the well-known results for matrices which are close to triangular ones.

Furthermore, due to inequality (3.3) matrix $A$ is unstable, provided

$$
\max _{k=1, \ldots, n} \operatorname{Re} a_{k k}-z_{n}(A)>0 .
$$

The latter result supplements the Rorhbach theorem MM, Chapter 3, Section 3.3.3].

\section{Operators with Hilbert-Schmidt Hermitian components}

In this section it is assumed that $A$ has the Hilbert-Schmidt imaginary component $A_{I} \equiv\left(A-A^{*}\right) / 2 i$ :

$$
N^{2}\left(A_{I}\right)=\text { Trace } A_{I}^{2}<\infty
$$

where $N($.$) is the Hilbert-Schmidt norm, again.$

Under (1.1), denote by $z_{H}(A)$ the unique non-negative root of the equation

$$
\nu(A) \sum_{k=0}^{\infty} \frac{\left(\sqrt{2} N\left(A_{I}\right)\right)^{k}}{\sqrt{k !} z^{k+1}}=1
$$

where $\nu(A)$ is defined by (1.4).

Theorem 4.1. Let relations (1.1), (1.2) and (4.1) hold. Then for any $\mu \in \sigma(D)$, there is a $\mu_{0} \in \sigma(A)$, such that $\left|\mu_{0}-\mu\right| \leq z_{H}(A)$. Moreover, relations (1.7)-(1.9) are true with $z_{H}(A)$ instead of $z(\phi)$. 
Proof. Condition (4.1) means that $A$ is quasi-Hermitian. That is, it is the sum of a self-adjoint operator and a compact one. So $A$ is simulteneously a quasinormal operator. Let us use the inequality

$$
\left\|(\lambda I-A)^{-1}\right\| \leq \sum_{k=0}^{\infty} \frac{\left(\sqrt{2} N\left(A_{I}\right)\right)^{k}}{\sqrt{k !} \rho^{k+1}(A, \lambda)}(\lambda \notin \sigma(A)) ;
$$

cf. Gi1, Theorem 3.4.2]. Now the required result is due to Theorem 1.1 and Corollary 1.2.

Further, for a constant $a>0$, the Schwarz inequality implies

$$
\left(\sum_{k=0}^{\infty} \frac{a^{k}}{\sqrt{k !}}\right)^{2}=\left(\sum_{k=0}^{\infty} \frac{2^{k / 2} a^{k}}{2^{k / 2} \sqrt{k !}}\right)^{2} \leq \sum_{j=0}^{\infty} 2^{-j} \sum_{k=0}^{\infty} \frac{2^{k} a^{2 k}}{k !}=2 \exp \left[2 a^{2}\right] .
$$

Now inequality (4.3) yields

$$
\left\|(\lambda I-A)^{-1}\right\| \leq \sqrt{2} \rho^{-1}(A, \lambda) \exp \left[\frac{2 N^{2}\left(A_{I}\right)}{\rho^{2}(A, \lambda)}\right] \quad(\lambda \notin \sigma(A)) .
$$

Hence, it follows that $z_{H}(A) \leq z_{1 H}(A)$ where $z_{1 H}(A)$ is the extreme right-hand (unique positive and simple) root of the equation

$$
\sqrt{2} \nu(A) z^{-1} \exp \left[2 z^{-2} N^{2}\left(A_{I}\right)\right]=1 .
$$

We need the following simple

Lemma 4.2. The unique positive root $z_{0}$ of the equation

$$
z e^{z}=a(a=\text { const }>0)
$$

satisfies the estimate

$$
z_{0} \geq \ln [1 / 2+\sqrt{1 / 4+a}] .
$$

If, in addition, the condition $a \geq e$ holds, then

$$
z_{0} \geq \ln a-\ln \ln a .
$$

Proof. Since $z \leq e^{z}-1(z \geq 0)$, we arrive at the relation $a \leq e^{2 z_{0}}-e^{z_{0}}$. Hence, $e^{z_{0}} \geq r_{1,2}$, where $r_{1,2}$ are the roots of the polynomial $y^{2}-y-a$. This proves inequality (4.8).

Furthermore, if the condition $a \geq e$ holds, then $z_{0} e^{z_{0}} \geq e$ and $z_{0} \geq 1$. Now (4.7) yields $e^{z_{0}} \leq a$ and $z_{0} \leq \ln a$. So $a=z_{0} e^{z_{0}} \leq e^{z_{0}} \ln a$. Hence, inequality (4.9) follows.

Clearly, (4.6) is equivalent to the equation

$$
2 \nu^{2}(A) z^{-2} \exp \left[4 z^{-2} N^{2}\left(A_{I}\right)\right]=1 .
$$

Denote $a_{H}(A) \equiv 2 N^{2}\left(A_{I}\right) \nu^{-2}(A)$ and substitute $z^{2}=4 N^{2}\left(A_{I}\right) x^{-1}$ in (4.10). Then we have $x e^{x}=a_{H}(A)$. Now Lemma 4.2 implies $z_{H}(A) \leq z_{1 H}(A) \leq \delta_{H}(A)$, where

$$
\delta_{H}(A)=\frac{2 N\left(A_{I}\right)}{\left[\ln \left(1 / 2+\sqrt{1 / 4+2 N^{2}\left(A_{I}\right) \nu^{-2}(A)}\right)\right]^{1 / 2}} .
$$

Clearly, $\delta_{H}(A) \rightarrow 0$, if either $V_{-} \rightarrow 0$ or $V_{+} \rightarrow 0$. 
Furthermore, Theorem 4.1 implies

Corollary 4.3. Let relations (1.1), (1.2) and (4.1) hold. Then for any $\mu \in \sigma(D)$, there is a $\mu_{0} \in \sigma(A)$, such that $\left|\mu-\mu_{0}\right| \leq \delta_{H}(A)$. Moreover, relations (1.7)-(1.9) hold with $\delta_{H}(A)$ instead of $z(\phi)$.

\section{Operators with Neumann-Schatten Hermitian components}

In this section it is assumed that the Hermitian component $A_{I}=\left(A-A^{*}\right) / 2 i$ belongs to the Neumann-Schatten ideal $C_{2 p}$ with some integer $p>1$ :

$$
N_{p}\left(A_{I}\right)=\left[\operatorname{Trace} A_{I}^{2 p}\right]^{1 / 2 p}<\infty .
$$

Here $N_{p}($.$) is the norm of ideal C_{2 p}$ :

$$
N_{p}(K)=\left[\operatorname{Trace}\left(K^{*} K\right)^{p}\right]^{1 / 2 p}\left(K \in C_{2 p}\right) .
$$

So $N_{1}()=.N($.$) is the Hilbert-Schmidt norm. Denote$

$$
\beta_{p}=2\left(1+\frac{2 p}{\exp (2 / 3) \ln 2}\right) \text { and } g_{p}(A)=\beta_{p} N_{p}\left(A_{I}\right)(p>1) .
$$

The constant $\beta_{p}$ shows the relation between $N_{p}(V)$ and $N_{p}\left(A_{I}\right)$, where $V$ is the nilpotent part of operator $A$ satisfying condition (5.1) (see [Gi1], Lemma 3.4.9).

Let $z_{p}(A)$ be the unique positive root of the equation

$$
\nu(A) \sum_{m=0}^{p-1} \sum_{k=0}^{\infty} \frac{g_{p}^{p k+m}(A)}{\sqrt{k !} z^{p k+m+1}}=1
$$

where $\nu(A)$ is defined by (1.4), again.

Theorem 5.1. Let relations (1.1), (1.2) and (5.1) hold. Then for any $\mu \in \sigma(D)$, there is a $\mu_{0} \in \sigma(A)$, such that $\left|\mu_{0}-\mu\right| \leq z_{p}(A)$. Moreover, inequalities (1.7)-(1.9) are true with $z_{p}(A)$ instead of $z(\phi)$.

Proof. Condition (5.1) means that $A$ is quasi-Hermitian. So it also is a quasinormal operator. Let us use the estimate

$$
\left\|(A-\lambda I)^{-1}\right\| \leq \sum_{m=0}^{p-1} \sum_{k=0}^{\infty} \frac{g_{p}^{k p+m}(A)}{\rho^{p k+m+1}(A, \lambda) \sqrt{k !}} \text { for all regular } \lambda ;
$$

see [Gi1, Theorem 3.4.8]. Now the required result is due to Theorem 1.1 and Corollary 1.2.

Due to inequalities (4.4) and (5.3),

$$
\left\|(A-\lambda I)^{-1}\right\| \leq \sqrt{2} \sum_{m=0}^{p-1} \frac{g_{p}^{m}(A)}{\rho^{m+1}(A, \lambda)} \exp \left[\frac{g_{p}^{2 p}(A)}{\rho^{2 p}(A, \lambda)}\right] .
$$

Hence, $z_{p}(A) \leq \tilde{z}_{p}(A)$ where $\tilde{z}_{p}(A)$ is the unique positive root of the equation

$$
\sqrt{2} \nu(A) \sum_{m=0}^{p-1} \frac{g_{p}^{m}(A)}{z^{m+1}} \exp \left[\frac{g_{p}^{2 p}(A)}{z^{2 p}}\right]=1 .
$$

Put

$$
c_{p}=\frac{g_{p}^{2 p}(A)}{2^{p} p ! \nu^{2 p}(A) p^{2 p-1}} .
$$


Lemma 5.2. With the notation

$$
\delta_{p}(A)=\frac{g_{p}(A)(2 p)^{1 / 2 p}}{\left[\ln \left[1 / 2+\sqrt{1 / 4+c_{p}}\right]^{1 / 2 p}\right.},
$$

the inequality $\tilde{z}_{p}(A) \leq \delta_{p}(A)$ is valid.

Proof. By the Hölder inequality

$$
\sum_{m=0}^{p-1} \frac{g_{p}^{m+1}(A)}{z^{m+1}} \leq l\left[\sum_{m=0}^{p-1} \frac{g_{p}^{2 p(m+1)}(A)}{z^{2 p(m+1)}}\right]^{1 / 2 p}
$$

where $l=p^{1-1 / 2 p}$. Thus,

$$
\sum_{m=0}^{p-1} \frac{g_{p}^{m+1}(A)}{z^{m+1}} \leq l\left[p ! \sum_{m=0}^{p-1} \frac{g_{p}^{2 p(m+1)}(A)}{m ! z^{2 p(m+1)}}\right]^{1 / 2 p} \leq l\left[p !\left(\exp \left[\frac{g_{p}^{2 p}(A)}{z^{2 p}}\right]-1\right)\right]^{1 / 2 p} .
$$

According to (5.5) with $z=\tilde{z}_{p}(A)$, we get

$$
\begin{gathered}
1=\sqrt{2} \nu(A) g_{p}^{-1} \sum_{m=0}^{p-1} \frac{g_{p}^{m+1}(A)}{z^{m+1}} \exp \left[\frac{g_{p}^{2 p}(A)}{2 z^{p}}\right] \\
\leq l \sqrt{2} \nu(A) g_{p}^{-1}\left[p !\left(\exp \left[\frac{g_{p}^{2 p}(A)}{z^{p}}\right]-1\right)\right]^{1 / 2 p} \exp \left[\frac{g_{p}^{2 p}(A)}{z^{2 p}}\right] .
\end{gathered}
$$

Hence

$$
1 \leq p !\left[l \sqrt{2} \nu(A) g_{p}^{-1}\right]^{2 p}(x-1) x=c_{p}^{-1}(x-1) x,
$$

where

$$
x=\exp \left[\frac{2 p g_{p}^{2 p}(A)}{z^{2 p}}\right] .
$$

Solving the inequality $c_{p}^{-1} x(x-1) \geq 1$, we can see that $x$ must be larger than both roots of the quadratic $x^{2}-x-c_{p}$. Thus,

$$
\exp \left[\frac{2 p g_{p}^{2 p}(A)}{z_{p}^{2 p}(A)}\right] \geq 1 / 2+\sqrt{1 / 4+c_{p}} .
$$

This proves the result.

Now the latter lemma and Theorem 5.1 imply

Corollary 5.3. Let relations (1.1), (1.2) and (5.1) hold. Then inequalities (1.7)(1.9) are true with $\delta_{p}(A)$ instead of $z(\phi)$.

\section{EXAMPLES}

6.1. Integral operators. Consider in $H=L^{2}[0,1]$ an integral operator $A$ defined by

$$
(A u)(x)=a(x) u(x)+\int_{0}^{1} K(x, s) u(s) d s\left(u \in L^{2}[0,1] ; x \in[0,1]\right)
$$

where $a($.$) is a real bounded measurable scalar-valued function, and K$ is a scalar Hilbert-Schmidt kernel:

$$
\int_{0}^{1} \int_{0}^{1}|K(x, s)|^{2} d s d x<\infty
$$


Take $(D u)(x)=a(x) u(x)$ and define $P(t)$ for $0 \leq t \leq 1$ by

$$
(P(t) u)(x)=0 \text { for } t<x \leq 1 \text { and }(P(t) u)(x)=u(x) \text { for } 0 \leq x<t .
$$

In addition, put $P(t)=I$ for $t>1$ and $P(t)=0$ for $t<0$. Then, relations (1.1) and (1.2) are valid with

$$
\left(V_{+} u\right)(x)=\int_{x}^{1} K(x, s) u(s) d s ;\left(V_{-} u\right)(x)=\int_{0}^{x} K(x, s) u(s) d s
$$

$\left(u \in L^{2}[0,1] ; x \in[0,1]\right)$. Moreover,

$$
N^{2}\left(V_{+}\right)=\int_{0}^{1} \int_{x}^{1}|K(x, s)|^{2} d s d x ; N^{2}\left(V_{-}\right)=\int_{0}^{1} \int_{0}^{x}|K(x, s)|^{2} d s d x .
$$

Clearly,

$$
N^{2}\left(A_{I}\right)=\int_{0}^{1} \int_{0}^{1}|K(x, s)-\bar{K}(s, x)|^{2} d s d x / 4 .
$$

According to (1.4) and (4.11), put $\nu(A)=\min \left\{N\left(V_{-}\right), N\left(V_{+}\right)\right\}$and

$$
\delta_{H}(A)=\frac{2 N\left(A_{I}\right)}{\left[\ln \left(1 / 2+\sqrt{1 / 4+2 N^{2}\left(A_{I}\right) \nu^{-2}(A)}\right)\right]^{1 / 2}} .
$$

Due to Corollary 4.3, for the integral operator (6.1), the following relations are true:

$$
\begin{gathered}
r_{s}(A) \geq \max \left\{0, \sup _{x \in[0,1]}|a(x)|-\delta_{H}(A)\right\}, \\
r_{l}(A) \leq \inf _{x \in[0,1]}|a(x)|+\delta_{H}(A) \text { and } \alpha(A) \geq \sup _{x \in[0,1]} a(x)-\delta_{H}(A) .
\end{gathered}
$$

About the upper bounds for the spectral radius of integral operators see, for instance, [Kr], Gi4, Gi5] and the references therein.

6.2. Matrix operators. Let $\left\{e_{k}\right\}_{k=1}^{\infty}$ be an orthogonal normed basis in $H$. Let $A$ be a linear operator in $H$ represented by a matrix with the entries

$$
a_{j k}=\left(A e_{k}, e_{j}\right)(j, k=1,2, \ldots),
$$

where (.,.) is the scalar product. Take $P(t)=\left\{P_{k}\right\}_{k=1}^{\infty}$, where $P_{k}$ are defined by

$$
P_{k}=\sum_{j=1}^{k}\left(., e_{j}\right) e_{j} .
$$

In the considered case $V_{+}, V_{-}$and $D$ are the upper triangular, lower triangular, and diagonal parts of $A$, respectively:

$$
\begin{gathered}
\left(V_{+} e_{k}, e_{j}\right)=a_{j k} \text { for } j<k,\left(V_{+} e_{k}, e_{j}\right)=0 \text { for } j>k, \\
\left(V_{-} e_{k}, e_{j}\right)=a_{j k} \text { for } j>k,\left(V_{-} e_{k}, e_{j}\right)=0 \text { for } j<k, \\
\left(D e_{k}, e_{k}\right)=a_{k k},\left(D e_{k}, e_{j}\right)=0 \text { for } j \neq k(j, k=1,2, \ldots) .
\end{gathered}
$$

Clearly, conditions (1.2) hold. Let the diagonal entries $a_{k k}(k=1,2, \ldots)$ be real and

$$
\sum_{j=1}^{\infty} \sum_{k=1, k \neq j}^{\infty}\left|a_{j k}\right|^{2}<\infty .
$$


Then $A_{I}$ and $V_{ \pm}$are Hilbert-Schmidt operators

$$
\begin{gathered}
N^{2}\left(A_{I}\right)=\sum_{j=1}^{\infty} \sum_{k=1}^{\infty}\left|a_{j k}-\bar{a}_{j k}\right|^{2} / 4<\infty, \\
N^{2}\left(V_{-}\right)=\sum_{j=1}^{\infty} \sum_{k=1}^{j-1}\left|a_{j k}\right|^{2}<\infty, N^{2}\left(V_{+}\right)=\sum_{j=1}^{\infty} \sum_{k=j+1}^{\infty}\left|a_{j k}\right|^{2}<\infty .
\end{gathered}
$$

Due to Corollary 4.3, for the matrix operator (6.3), the following relations are true:

$$
\begin{gathered}
r_{s}(A) \geq \max \left\{0, \sup _{k}\left|a_{k k}\right|-\delta_{H}(A)\right\}, \\
r_{l}(A) \leq \inf _{k}\left|a_{k k}\right|+\delta_{H}(A) \text { and } \alpha(A) \geq \sup _{k} a_{k k}-\delta_{H}(A)
\end{gathered}
$$

where $\delta_{H}(A)$ is defined by (6.2). So matrix $A$ is unstable, provided $\max _{k} a_{k k}-$ $\delta_{H}(A) \geq 0$. For non-negative matrices the following estimate is well known $\mathrm{Kr}$. inequality (16.15)]:

$$
r_{s}(A) \geq \tilde{r}_{\infty}(A) \equiv \min _{j=1, \ldots, \infty} \sum_{k=1}^{\infty} a_{j k} .
$$

Relation (6.4) improves estimate (6.6) in the case $\left|a_{j k}\right|=a_{j k}(j, k=1,2, \ldots)$ provided $\max _{k} a_{k k}-\delta_{H}(A)>\tilde{r}_{\infty}(A)$. That is, (6.4) is sharper than (6.6) for matrices which are close to triangular ones, since $\delta_{H}(A) \rightarrow 0$ when $V_{-} \rightarrow 0$ or $V_{+} \rightarrow 0$.

\section{REFERENCES}

[Br] Brodskii, M.S. Triangular and Jordan Representations of Linear Operators, Transl. Math. Monogr., v. 32, Amer. Math. Soc. Providence, R. I. 1971. MR 48:904

[Gi1] Gil', M.I. Norm Estimations for Operator-valued Functions and Applications. Marcel Dekker, Inc. New York, 1995. MR 97h:47002

[Gi2] Gil', M.I. A nonsingularity criterion for matrices, Linear Algebra Appl. 253, (1997) 79-87. MR 97m:15003

[Gi3] Gil', M.I. Stability of Finite and Infinite Dimensional Systems, Kluwer Ac. Publishers, Boston-Dordrecht-London, 1998. MR 99m:34110

[Gi4] Gil', M.I. Invertibility conditions and bounds for spectra of matrix integral operators, Monatshefte für Mathematik 129, (2000) 15-24. MR 2000m:47062

[Gi5] Gil', M.I. Invertibility and positive invertibility conditions of integral operators in $L^{\infty}, J$. of Integral Equations and Appl. 13 (2001), 1-14. MR 2002c:47109

[Gi6] Gil', M.I. On invertibility and positive invertibility of matrices, Linear Algebra Appl. 327, (2001), 95-104. MR 2002b:15006

[GK] Gohberg, I. and Krein, M.G. Theory and Applications of Volterra Operators in Hilbert Space, Trans. Mathem. Monographs, vol. 24, Amer. Math. Soc., R.I., 1970. MR 41:9041

[Ko] König, H. Eigenvalue Distribution of Compact Operators, Birkhäuser Verlag, Basel- BostonStuttgart, 1986. MR 88j:47021

[Kr] Krasnosel'skii, M.A., Lifshits, J. and Sobolev A. Positive Linear Systems. The Method of Positive Operators, Heldermann Verlag, Berlin, 1989. MR 91f:47051

[MM] Marcus, M. and Minc, H. A Survey of Matrix Theory and Matrix Inequalities, Allyn and Bacon, Boston, 1964. MR 29:112

[Pi] Pietsch, A. Eigenvalues and s-Numbers, Cambridge University Press, Cambridge, 1987. MR 88j:47022b

Department of Mathematics, Ben Gurion University of the Negev, P.O. Box 653, BeER-SHeva 84105, IsRAel

E-mail address: gilmi@black.bgu.ac.il 